\title{
Entropy dissipation estimates for inhomogeneous zero-range processes
}

\author{
Jonathan Hermon, Justin Salez
}

March 5, 2019

\begin{abstract}
Introduced by Lu \& Yau (CMP, 1993), the martingale decomposition method is a powerful recursive strategy that has produced sharp log-Sobolev inequalities for homogeneous particle systems. However, the intractability of certain covariance terms has so far precluded applications to heterogeneous models. Here we demonstrate that the existence of an appropriate coupling can be exploited to bypass this limitation effortlessly. Our main result is a dimension-free modified log-Sobolev inequality for zero-range processes on the complete graph, under the only requirement that all rate increments lie in a compact subset of $(0, \infty)$. This settles an open problem raised by Caputo \& Posta (PTRF, 2007) and reiterated by Caputo, Dai Pra \& Posta (AIHP, 2009). We believe that our approach is simple enough to be applicable to many systems.
\end{abstract}

\section{Contents}

1 Introduction

1.1 Entropy dissipation estimates $\ldots \ldots \ldots \ldots \ldots \ldots$

1.2 Zero-range dynamics . . . . . . . . . . . . . . . . . . .

1.3 Result and related works $\ldots \ldots \ldots \ldots \ldots \ldots$

\section{Proofs}

2.1 Monotone coupling . . . . . . . . . . . . . . . .

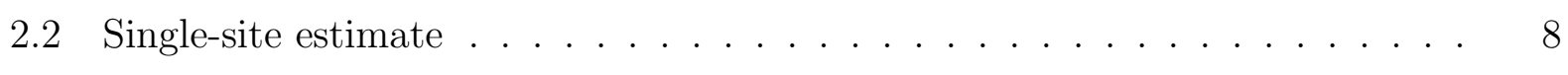

2.3 The induction argument ......................... 10 


\section{Introduction}

\subsection{Entropy dissipation estimates}

Consider a reversible Markov generator $Q$ with respect to some probability distribution $\pi$ on a finite state space $\Omega$. In other words, $Q$ is a $\Omega \times \Omega$ matrix with non-negative off-diagonal entries, with each row summing up to 0 , and satisfying the local balance equations

$$
\pi(x) Q(x, y)=\pi(y) Q(y, x),
$$

for all $x, y \in \Omega$. When $Q$ is irreducible, the Markov semi-group $P_{t}=e^{t Q}$ generated by $Q$ mixes: for any observable $f: \Omega \rightarrow(0, \infty)$, we have the pointwise convergence

$$
P_{t} f \underset{t \rightarrow \infty}{\longrightarrow} \mathbb{E}[f(X)],
$$

where throughout the paper, $X$ denotes a $\pi$-distributed random variable. A natural way to quantify this convergence consists in measuring the rate at which the entropy

$$
\operatorname{Ent}(f):=\mathbb{E}[f(X) \log f(X)]-\mathbb{E}[f(X)] \log \mathbb{E}[f(X)],
$$

decays along the semi-group. Specifically, one looks for a constant $\alpha>0$, as large as possible, such that for all observables $f: \Omega \rightarrow(0, \infty)$ and all times $t \geq 0$,

$$
\operatorname{Ent}\left(P_{t} f\right) \leq e^{-\alpha t} \operatorname{Ent}(f) .
$$

The optimal value of $\alpha$ is called the entropy dissipation constant and will be denoted by $\alpha(Q)$. Writing $\mathcal{E}(f, g):=-\mathbb{E}[f(X)(Q g)(X)]$ for the underlying Dirichlet form, we compute

$$
\frac{d}{d t} \operatorname{Ent}\left(P_{t} f\right)=-\mathcal{E}\left(P_{t} f, \log P_{t} f\right) .
$$

Thus, $\alpha(Q)$ is more effectively characterized as the largest constant $\alpha>0$ such that the following modified log-Sobolev inequality (MLSI) holds: for all observables $f: \Omega \rightarrow(0, \infty)$,

$$
\mathcal{E}(f, \log f) \geq \alpha \operatorname{Ent}(f) .
$$

We refer to the tutorial paper [2] or the textbook 21] for more details on this fundamental functional inequality and its relation to hypercontractivity, concentration and mixing times. For intrinsic reasons exposed in [20, establishing sharp MLSI's for Markov chains on finite spaces remains a notoriously challenging task. A natural and important context where such entropic estimates have received a particular attention is that of interacting particle systems and, in particular, zero-range processes. 


\subsection{Zero-range dynamics}

Introduced by Spitzer [23], the zero-range process (ZRP) is a generic conservative particle system in which individual jumps occur at a rate which only depends on the source, the destination, and the number of particles present at the source. We shall here focus on the mean-field version of the model, which is parameterized by the following ingredients:

- two integers $n, m \geq 1$ representing the numbers of sites and particles, respectively;

- a probability vector $p=\left(p_{1}, \ldots, p_{n}\right)$ specifying the distribution of the jumps;

- a function $r_{i}:\{1,2, \ldots\} \rightarrow(0, \infty)$ encoding the kinetics at site $i \in[n]:=\{1, \ldots, n\}$.

The ZRP with these parameters is a continuous-time Markov chain on the state space

$$
\Omega:=\left\{x \in \mathbb{Z}_{+}^{n}: \sum_{i=1}^{n} x_{i}=m\right\}
$$

where $x_{i}$ represents the number of particles at site $i$. The action of the generator is given by

$$
(Q f)(x):=\sum_{i=1}^{n} \sum_{j=1}^{n} r_{i}\left(x_{i}\right) p_{j}\left(f\left(x+\delta_{j}-\delta_{i}\right)-f(x)\right),
$$

where $\left(\delta_{1}, \ldots, \delta_{n}\right)$ denotes the canonical $n$-dimensional basis, and with the convention that $r_{i}(0)=0$ for all $i \in[n]$ (no jump from empty sites). This generator is easily seen to be reversible with respect to the explicit distribution

$$
\pi(x) \propto \prod_{i=1}^{n} \frac{p_{i}^{x_{i}}}{r_{i}(1) \cdots r_{i}\left(x_{i}\right)}
$$

with the normalization being chosen so that $\pi$ is a probability distribution on $\Omega$. A somewhat degenerate but instructive situation is obtained with the linear choice

$$
r_{i}(\ell)=c \times \ell
$$

for all $i \in[n]$ and $\ell \geq 0$, where $c>0$ is an arbitrary constant. In this case, the model trivializes in the sense that the $m$ particles perform independent random walks, each jumping at rate $c$ according to $p$. Thanks to the tensorization property of variance and entropy, the Poincaré and modified log-Sobolev constants of the whole system then reduce to those of a single particle, which are easily seen to be bounded away from 0 independently of $p$. In light 
of this, it is reasonable to expect that dimension-free functional inequalities will persist in the perturbative regime where the rate functions are nearly linear. Turning this intuition into rigorous estimates has been and continues to be a subject of active research, see e.g., [17, 14, 4, 3, 22, 12] on the Poincaré side, and [8, 6, 11, 5, 9] on the log-Sobolev side.

\subsection{Result and related works}

Perhaps the most natural way to formalize the idea that the rate functions should be nearly linear consists in requiring that their increments all lie in a fixed compact subset of $(0, \infty)$.

Assumption 1. There are constants $\Delta, \delta>0$ such that for every $i \in[n]$ and every $\ell \in \mathbb{Z}_{+}$,

$$
\delta \leq r_{i}(\ell+1)-r_{i}(\ell) \leq \Delta
$$

In a remarkable work [5], Caputo, Dai Pra \& Posta developed a novel method, based on the so-called Bochner-Bakry-Emery approach introduced in [1], to establish a dimension-free MLSI in the perturbative regime where the ratio $\Delta / \delta$ is sufficiently small.

Theorem 1 (Caputo, Dai Pra \& Posta, [5]). Suppose that Assumption 1 holds with

$$
\frac{\Delta}{\delta}<2
$$

and take $p=\left(\frac{1}{n}, \ldots, \frac{1}{n}\right)$. Then, for any number $m$ of particles, the ZRP satisfies

$$
\alpha(Q) \geq 2 \delta-\Delta
$$

In the so-called homogeneous setting where the rate function $r_{i}$ is not allowed to depend on $i$, Assumption 1 can be relaxed at the price of considerable efforts, see [6]. However, the martingale approach used therein does not extend to inhomogeneous models in a natural way and, to the best of our knowledge, Theorem 1 constitutes the only available criterium for inhomogeneous rates. Although the bound (13) trivializes as $\Delta$ approaches the threshold $2 \delta$, the authors predicted the persistence of a dimension-free MLSI beyond the perturbative regime (12). Specifically, they formulated the following conjecture, reiterated in [6].

Conjecture 1 (Caputo, Dai Pra \& Posta, [5]). Under Assumption 1, there is a dimensionfree constant $c(\delta, \Delta)>0$ such that for $p=\left(\frac{1}{n}, \ldots, \frac{1}{n}\right)$ and any number $m$ of particles,

$$
\alpha(Q) \geq c(\delta, \Delta)
$$


Proving this requires new ideas, since it was noted in [5] that the convexity of $t \mapsto$ $\operatorname{Ent}\left(P_{t} f\right)$ fails as $\frac{\Delta}{\delta}$ gets large, making the Bochner-Bakry-Emery approach unapplicable. In the present paper, we establish the following strengthening of Conjecture 1.

Theorem 2 (Dimension-free MLSI). Under the sole Assumption 1, and for any choice of the parameters $p$ and $m$, the modified log-Sobolev constant of the ZRP satisfies

$$
\alpha(Q) \geq \frac{\delta^{2}}{2 \Delta} .
$$

This estimate is sharp up to a factor 2, as can already been seen in the linear case (10) (see, [2, Example 3.10]). More importantly, our main contribution lies in the simplicity of the method used to prove Theorem 2. Our starting point is the following elementary observation, which is a straightforward consequence of the product form (9).

Observation 1 (Recursive structure). If $X=\left(X_{1}, \ldots, X_{n}\right)$ has law $\pi$, then for any site $i \in$ $[n]$, the conditional law of $\left(X_{1}, \ldots, X_{i-1}, X_{i+1}, \ldots, X_{n}\right)$ given $X_{i}$ coincides with the stationary law of a new ZRP with $n-1$ sites, $m-X_{i}$ particles, rates $\left(r_{1}, \ldots, r_{i-1}, r_{i+1}, \ldots, r_{n}\right)$ and probability vector $\left(\frac{p_{1}}{1-p_{i}}, \ldots, \frac{p_{i-1}}{1-p_{i}}, \frac{p_{i+1}}{1-p_{i}}, \ldots, \frac{p_{n}}{1-p_{i}}\right)$.

Since the new ZRP inherits Assumption 1 from the original one, a natural approach to Theorem 2 consists in proceeding by induction over the dimension $n$. This is in fact a classical strategy for establishing functional inequalities, known as the martingale decomposition method. Introduced by Lu \& Yau [19] in the context of Kawasaki and Glauber dynamics, it has been successfully applied to various interacting particle systems [24, 18, 10, 6], as well as other Markov chains enjoying an appropriate recursive structure [7, 15, 16, 13]. To convert the above observation into an effective functional inequality however, one needs to estimate the Dirichlet form of the $n$-dimensional system in terms of that of the $(n-1)$-dimensional system. Because of the interaction between particles, this decomposition inevitably produces certain cross terms, whose intractibility has so far precluded applications to heterogeneous models. Our main contribution consists in showing that the existence of an appropriate coupling - see Proposition 1 below - allows one to bypass this limitation effortlessly. We firmly believe that our idea is simple enough to be applicable to many other settings.

\section{Proofs}

From now onwards, we suppose that Assumption 1 is satisfied. 


\subsection{Monotone coupling}

Using the fact that each rate function $r_{i}:\{1,2, \ldots\} \rightarrow(0, \infty)$ is non-decreasing, we may enrich our ZRP by adding a tagged particle on top of it, whose position $(J(t): t \geq 0)$ evolves as follows: conditionally on the background process $(X(t): t \geq 0)$ being currently in some state $x \in \Omega$, we let the tagged particle jump across $[n]$ according to the Markov generator

$$
L_{x}(i, j):=\left(r_{i}\left(x_{i}+1\right)-r_{i}\left(x_{i}\right)\right)\left(p_{j}-\mathbf{1}_{(\mathbf{i}=\mathbf{j})}\right) \text {. }
$$

In more formal terms, we consider a Markov process $((X(t), J(t)): t \geq 0)$ taking values in the product space $\widehat{\Omega}:=\Omega \times[n]$, and evolving according to the generator

$$
\widehat{Q}((x, i),(y, j)):=Q(x, y) \mathbf{1}_{(i=j)}+L_{x}(i, j) \mathbf{1}_{(x=y)}
$$

We refer to this process as the tagged ZRP. An elementary but crucial observation is that

(i) the first-coordinate $(X(t): t \geq 0)$ is a ZRP with parameters $(n, m, p, r)$;

(ii) the aggregated process $\left(X(t)+\delta_{J(t)}: t \geq 0\right)$ is a ZRP with parameters $(n, m+1, p, r)$.

The existence of such a monotone coupling between zero-range processes with different numbers of particles is of course well known, and has been extensively used in the past. However, its consequences on the martingale approach do not seem to have been explored, and this is where our conceptual contribution lies. Specifically, our interest will here reside in the invariant law $\widehat{\pi}$ of $\widehat{Q}$, which is uniquely determined by the global balance equations

$$
\sum_{x \in \Omega} \widehat{\pi}(x, j) Q(x, y)+\sum_{i=1}^{n} \widehat{\pi}(y, i) L_{y}(i, j)=0
$$

for all $(y, j) \in \widehat{\Omega}$. Although we do not have any explicit expression for $\widehat{\pi}$, we note that its first marginal has to be invariant under $Q$ by construction, and is therefore simply the law $\pi$ defined at (9). We also note that, in the linear case (10), the generator $L_{x}$ of the tagged particle becomes independent of the background state $x \in \Omega$, resulting in the product form

$$
\widehat{\pi}(x, j)=\pi(x) p_{j}
$$

The next lemma states that under Assumption 1, $\widehat{\pi}$ is not far from this product measure.

Lemma 2 (Product-measure approximation). The invariant law of the tagged ZRP satisfies

$$
\max _{(x, j) \in \widehat{\Omega}}\left\{\frac{\widehat{\pi}(x, j)}{\pi(x) p_{j}}\right\} \leq \frac{\Delta}{\delta} .
$$


Proof. Fix $j \in[n]$ and choose $y \in \Omega$ such that

$$
\frac{\widehat{\pi}(y, j)}{\pi(y)}=\max _{x \in \Omega}\left\{\frac{\widehat{\pi}(x, j)}{\pi(x)}\right\} .
$$

At the point $(y, j)$, the first sum in the global balance equation (18) is non-positive. Indeed,

$$
\sum_{x \in \Omega} \widehat{\pi}(x, j) Q(x, y) \leq \frac{\widehat{\pi}(y, j)}{\pi(y)} \sum_{x \in \Omega} \pi(x) Q(x, y)=0
$$

where the equality is simply the balance equation $\pi Q=0$ at state $y$. Consequently, the second sum in (18) must be non-negative. In other words,

$$
\begin{aligned}
0 & \leq \sum_{i=1}^{n} \widehat{\pi}(y, i) L_{y}(i, j) \\
& \leq \sum_{i=1}^{n} \widehat{\pi}(y, i)\left(\Delta p_{j}-\delta \mathbf{1}_{(i=j)}\right)=\Delta p_{j} \pi(y)-\delta \widehat{\pi}(y, j),
\end{aligned}
$$

where we have used Assumption (1), and then the fact that the first marginal of $\widehat{\pi}$ is $\pi$.

These considerations lead us to the following result, which will constitute our main tool.

Proposition 1 (Main coupling). Given a site $i \in[n]$, we may jointly construct a $\pi$-distributed random variable $X$ and $a[n] \backslash\{i\}$-valued random variable $J$ in such a way that

$$
\operatorname{Law}\left(X-\delta_{i}+\delta_{J} \mid X_{i}=\ell\right)=\operatorname{Law}\left(X \mid X_{i}=\ell-1\right)
$$

for each level $\ell \in\{1, \ldots, m\}$ and moreover, for every $(x, j) \in \Omega \times([n] \backslash\{i\})$,

$$
\mathbb{P}(J=j \mid X=x) \leq \frac{\Delta p_{j}}{\delta\left(1-p_{i}\right)} .
$$

Proof. We simply let $X_{i}$ be distributed according to the $i$-th marginal of $\pi$ and, given that $X_{i}=\ell$, we let $\left(\left(X_{1}, \ldots, X_{i-1}, X_{i+1}, \ldots, X_{n}\right), J\right)$ be distributed according to the stationary distribution of the $(n-1)$-dimensional tagged ZRP with $m-\ell$ particles, rates $\left(r_{1}, \ldots, r_{i-1}, r_{i+1}, \ldots, r_{n}\right)$ and probability vector $\left(\frac{p_{1}}{1-p_{i}}, \ldots, \frac{p_{i-1}}{1-p_{i}}, \frac{p_{i+1}}{1-p_{i}}, \ldots, \frac{p_{n}}{1-p_{i}}\right)$. Observation 1 ensures that the random variable $X:=\left(X_{1}, \ldots, X_{n}\right)$ is distributed according to $\pi$. Moreover, property (24) is guaranteed by Lemma 2, Finally, the identity (23) is clear since under both distributions, the $i$-th coordinate is almost-surely equal to $\ell-1$ while the joint law of the remaining coordinates is the same, thanks to observation (ii) above. 


\subsection{Single-site estimate}

In this section, we show how the above coupling implies - without effort - the crucial local MLSI needed for our inductive proof of Theorem 2. We start by introducing some notation. Let us decompose the Dirichlet form of the process as

$$
\mathcal{E}(f, g)=\frac{1}{2} \sum_{i=1}^{n} \sum_{j=1}^{n} \mathcal{E}_{i j}(f, g)
$$

where $\mathcal{E}_{i j}(f, g)$ captures the contribution from all jumps with source $i$ and destination $j$, i.e.

$$
\mathcal{E}_{i j}(f, g):=p_{j} \mathbb{E}\left[r_{i}\left(X_{i}\right)\left(f\left(X-\delta_{i}+\delta_{j}\right)-f(X)\right)\left(g\left(X-\delta_{i}+\delta_{j}\right)-g(X)\right)\right] .
$$

Given an observable $f: \Omega \rightarrow(0, \infty)$ and a site $i \in[n]$, we define $f_{i}:\{0, \ldots, m\} \rightarrow(0, \infty)$ by

$$
f_{i}(\ell):=\mathbb{E}\left[f(X) \mid X_{i}=\ell\right]
$$

With this notation in hands, our aim is to establish the following local estimate, which relates the Dirichlet contribution from a single site $i$ to the conditional entropy given $X_{i}$.

Proposition 2 (Local MLSI). For any observable $f: \Omega \rightarrow(0, \infty)$ and any site $i \in[n]$,

$$
\mathbb{E}\left[f_{i}\left(X_{i}\right) \log f_{i}\left(X_{i}\right)\right]-\mathbb{E}[f(X)] \log \mathbb{E}[f(X)] \leq \frac{\Delta}{\delta^{2}} \sum_{j \in[n] \backslash\{i\}} \mathcal{E}_{i j}(f, \log f) .
$$

Proof. Consider the bivariate function $\mathcal{H}:(0, \infty)^{2} \rightarrow[0, \infty)$ defined by

$$
\mathcal{H}(u, v):=(u-v)(\log u-\log v) .
$$

This function is convex, because its hessian matrix

$$
\left[\begin{array}{cc}
\partial_{u u} \mathcal{H} & \partial_{u v} \mathcal{H} \\
\partial_{v u} \mathcal{H} & \partial_{v v} \mathcal{H}
\end{array}\right]=\frac{u+v}{(u v)^{2}}\left[\begin{array}{cc}
v^{2} & -u v \\
-u v & u^{2}
\end{array}\right]
$$

is positive semi-definite at each point $(u, v) \in(0, \infty)^{2}$. Now, fix a site $i \in[n]$, and let $(X, J)$ be as in Proposition 1. On the event $\left\{X_{i} \geq 1\right\}$, the definition of $f_{i}$ and Property (23) imply

$$
\begin{aligned}
f_{i}\left(X_{i}\right) & =\mathbb{E}\left[f(X) \mid X_{i}\right], \\
f_{i}\left(X_{i}-1\right) & =\mathbb{E}\left[f\left(X+\delta_{J}-\delta_{i}\right) \mid X_{i}\right] .
\end{aligned}
$$


Thus, the pair $\left(f_{i}\left(X_{i}\right), f_{i}\left(X_{i}-1\right)\right)$ is the conditional expectation of $\left(f(X), f\left(X-\delta_{i}+\delta_{J}\right)\right)$ given $X_{i}$. By the conditional Jensen inequality, we deduce that on the event $\left\{X_{i} \geq 1\right\}$,

$$
\mathcal{H}\left(f_{i}\left(X_{i}\right), f_{i}\left(X_{i}-1\right)\right) \leq \mathbb{E}\left[\mathcal{H}\left(f(X), f\left(X-\delta_{i}+\delta_{J}\right)\right) \mid X_{i}\right]
$$

Multiplying by $\left(1-p_{i}\right) r_{i}\left(X_{i}\right)$ and taking expectations, we obtain

$$
\begin{aligned}
\left(1-p_{i}\right) \mathbb{E}\left[r_{i}\left(X_{i}\right) \mathcal{H}\left(f_{i}\left(X_{i}\right), f_{i}\left(X_{i}-1\right)\right)\right] & \leq\left(1-p_{i}\right) \mathbb{E}\left[r_{i}\left(X_{i}\right) \mathcal{H}\left(f(X), f\left(X-\delta_{i}+\delta_{J}\right)\right)\right] \\
& \leq \frac{\Delta}{\delta} \sum_{j \in[n] \backslash\{i\}} \mathcal{E}_{i j}(f, \log f),
\end{aligned}
$$

where the second line uses Property (24). Thus, our task boils down to proving

$$
\mathbb{E}\left[f_{i}\left(X_{i}\right) \log f_{i}\left(X_{i}\right)\right]-\mathbb{E}[f(X)] \log \mathbb{E}[f(X)] \leq \frac{\left(1-p_{i}\right)}{\delta} \mathbb{E}\left[r_{i}\left(X_{i}\right) \mathcal{H}\left(f_{i}\left(X_{i}\right), f_{i}\left(X_{i}-1\right)\right)\right]
$$

Since $\mathbb{E}\left[f_{i}\left(X_{i}\right)\right]=\mathbb{E}[f(X)]$, the left-hand side is the entropy of $f_{i}$ with respect to the law of $X_{i}$. On the other hand, the right-hand side is exactly $\frac{1}{\delta} \mathcal{E}_{K}\left(f_{i}, \log f_{i}\right)$, where $\mathcal{E}_{K}$ denotes the Dirichlet form of the birth-and-death generator $K$ on $\{0, \ldots, m\}$ defined by

$$
\begin{aligned}
\forall \ell \in\{1, \ldots, m\}, & K(\ell, \ell-1) & :=\left(1-p_{i}\right) r_{i}(\ell) \\
\forall \ell \in\{0, \ldots, m-1\}, & K(\ell, \ell+1) & :=p_{i} \sum_{j \in[n] \backslash\{i\}} \mathbb{E}\left[r_{j}\left(X_{j}\right) \mid X_{i}=\ell\right] .
\end{aligned}
$$

Note that these rates are reversible with respect to the law of $X_{i}$, because they mimic the zero-range dynamics at site $i$. In terms of the generator $K$, the above claim reads

$$
\alpha(K) \geq \delta
$$

It remains to establish this one-dimensional MLSI. By Assumption 1, the death rates satisfy

$$
K(\ell+1, \ell)-K(\ell, \ell-1)=\left(1-p_{i}\right)\left(r_{i}(\ell+1)-r_{i}(\ell)\right) \geq\left(1-p_{i}\right) \delta
$$

Regarding the birth rates, we may invoke Property (23) to write

$$
K(\ell-1, \ell)-K(\ell, \ell+1)=p_{i} \sum_{j \in[n] \backslash\{i\}} \mathbb{E}\left[r_{j}\left(X_{j}+\mathbf{1}_{(J=j)}\right)-r_{j}\left(X_{j}\right) \mid X_{i}=\ell\right] \geq p_{i} \delta
$$

For birth-and-death chains, these uniform bounds on the rate increments are known to imply the MLSI (35), see e.g., [5, Theorem 3.1] or [6, Lemma 2.2]. 


\subsection{The induction argument}

We are finally in position to prove Theorem 2 by induction over $n$. The claim is trivial for $n=1$. We now assume that $n \geq 2$ and that the claim is already proven for $(n-$ 1)-dimensional systems. Fix an observable $f: \Omega \rightarrow(0, \infty)$ and a site $i \in[n]$, and consider the $(n-1)$-dimensional ZRP obtained by conditioning on $X_{i}$, as in Observation 1. Viewing $f$ as a function of the $n-1$ remaining variables, the induction hypothesis ensures that

$$
\operatorname{Ent}\left(f \mid X_{i}\right) \leq \frac{2 \Delta}{\delta^{2}} \sum_{j, k \in[n] \backslash\{i\}} \frac{p_{j}}{1-p_{i}} \mathbb{E}\left[r_{k}\left(X_{k}\right) \mathcal{H}\left(f(X), f\left(X+\delta_{j}-\delta_{k}\right)\right) \mid X_{i}\right],
$$

where the entropy on the left-hand side is computed conditionally on $X_{i}$, i.e.

$$
\operatorname{Ent}\left(f \mid X_{i}\right):=\mathbb{E}\left[f(X) \log f(X) \mid X_{i}\right]-f_{i}\left(X_{i}\right) \log f_{i}\left(X_{i}\right) .
$$

Taking expectations, we arrive at

$$
\mathbb{E}[f(X) \log f(X)] \leq \mathbb{E}\left[f_{i}\left(X_{i}\right) \log f_{i}\left(X_{i}\right)\right]+\frac{\Delta}{\delta^{2}\left(1-p_{i}\right)} \sum_{j, k \in[n] \backslash\{i\}} \mathcal{E}_{j k}(f, \log f) .
$$

We now multiply by $1-p_{i}$ and use Proposition 2 to obtain

$$
\begin{aligned}
\left(1-p_{i}\right) \operatorname{Ent}(f) & \leq \frac{\Delta}{\delta^{2}} \sum_{j \in[n] \backslash\{i\}}\left(\left(1-p_{i}\right) \mathcal{E}_{i j}(f, \log f)+\sum_{k \in[n] \backslash\{i\}} \mathcal{E}_{k j}(f, \log f)\right) \\
& \leq \frac{\Delta}{\delta^{2}} \sum_{j \in[n] \backslash\{i\}} \sum_{k=1}^{n} \mathcal{E}_{k j}(f, \log f) .
\end{aligned}
$$

Summing over all sites $i \in[n]$ and recalling (25), we conclude that

$$
(n-1) \operatorname{Ent}(f) \leq \frac{2 \Delta}{\delta^{2}}(n-1) \mathcal{E}(f, \log f) .
$$

Since this is true for every $f: \Omega \rightarrow(0, \infty)$, we have just established a MLSI with constant $\frac{\delta^{2}}{2 \Delta}$ for our $n$-dimensional ZRP. This completes our induction step.

\section{References}

[1] D. Bakry and Michel Émery. Diffusions hypercontractives. In Séminaire de probabilités, XIX, 1983/84, volume 1123 of Lecture Notes in Math., pages 177-206. Springer, Berlin, 1985. 
[2] Sergey G. Bobkov and Prasad Tetali. Modified logarithmic Sobolev inequalities in discrete settings. J. Theoret. Probab., 19(2):289-336, 2006.

[3] Anne-Severine Boudou, Pietro Caputo, Paolo Dai Pra, and Gustavo Posta. Spectral gap estimates for interacting particle systems via a Bochner-type identity. J. Funct. Anal., 232(1):222-258, 2006. MR2200172.

[4] Pietro Caputo. Spectral gap inequalities in product spaces with conservation laws. In Stochastic analysis on large scale interacting systems, volume 39 of Adv. Stud. Pure Math., pages 53-88. Math. Soc. Japan, Tokyo, 2004. MR2073330.

[5] Pietro Caputo, Paolo Dai Pra, and Gustavo Posta. Convex entropy decay via the Bochner-Bakry-Emery approach. Ann. Inst. Henri Poincaré Probab. Stat., 45(3):734$753,2009$.

[6] Pietro Caputo and Gustavo Posta. Entropy dissipation estimates in a zero-range dynamics. Probab. Theory Related Fields, 139(1-2):65-87, 2007. MR2322692.

[7] Paolo Dai Pra, Anna Maria Paganoni, and Gustavo Posta. Entropy inequalities for unbounded spin systems. Ann. Probab., 30(4):1959-1976, 2002.

[8] Paolo Dai Pra and Gustavo Posta. Logarithmic Sobolev inequality for zero-range dynamics. Ann. Probab., 33(6):2355-2401, 2005. MR2184099.

[9] Max Fathi and Jan Maas. Entropic Ricci curvature bounds for discrete interacting systems. Ann. Appl. Probab., 26(3):1774-1806, 2016.

[10] Fuqing Gao and Jeremy Quastel. Exponential decay of entropy in the random transposition and Bernoulli-Laplace models. Ann. Appl. Probab., 13(4):1591-1600, 2003.

[11] Benjamin T. Graham. Rate of relaxation for a mean-field zero-range process. Ann. Appl. Probab., 19(2):497-520, 2009. MR2521877.

[12] Jonathan Hermon and Justin Salez. A version of Aldous' spectral-gap conjecture for the zero range process. ArXiv e-prints, August 2018.

[13] Jonathan Hermon and Justin Salez. Modified log-Sobolev inequalities for strongRayleigh measures. arXiv e-prints, page arXiv:1902.02775, Feb 2019. 
[14] E. Janvresse, C. Landim, J. Quastel, and H. T. Yau. Relaxation to equilibrium of conservative dynamics. I. Zero-range processes. Ann. Probab., 27(1):325-360, 1999. MR1681098.

[15] Mark Jerrum and Jung-Bae Son. Spectral gap and log-sobolev constant for balanced matroids. In The 43rd Annual IEEE Symposium on Foundations of Computer Science, 2002. Proceedings., pages 721-729, Nov 2002.

[16] Mark Jerrum, Jung-Bae Son, Prasad Tetali, and Eric Vigoda. Elementary bounds on Poincaré and log-Sobolev constants for decomposable Markov chains. Ann. Appl. Probab., 14(4):1741-1765, 2004.

[17] C. Landim, S. Sethuraman, and S. Varadhan. Spectral gap for zero-range dynamics. Ann. Probab., 24(4):1871-1902, 1996. MR1415232.

[18] Tzong-Yow Lee and Horng-Tzer Yau. Logarithmic Sobolev inequality for some models of random walks. Ann. Probab., 26(4):1855-1873, 1998.

[19] Sheng Lin Lu and Horng-Tzer Yau. Spectral gap and logarithmic Sobolev inequality for Kawasaki and Glauber dynamics. Comm. Math. Phys., 156(2):399-433, 1993.

[20] Laurent Miclo. Some drawbacks of finite modified logarithmic Sobolev inequalities. Math. Scand., 123(1):147-159, 2018.

[21] Ravi Montenegro and Prasad Tetali. Mathematical aspects of mixing times in Markov chains. Found. Trends Theor. Comput. Sci., 1(3):x+121, 2006. MR2341319.

[22] Ben Morris. Spectral gap for the zero range process with constant rate. Ann. Probab., 34(5):1645-1664, 2006. MR2271475.

[23] Frank Spitzer. Interaction of Markov processes. Advances in Math., 5:246-290 (1970), 1970. MR0268959.

[24] Horng-Tzer Yau. Logarithmic Sobolev inequality for generalized simple exclusion processes. Probab. Theory Related Fields, 109(4):507-538, 1997. 